\title{
High dietary protein intake induces endothelial dysfunction in uninephrectomized rats
}

\author{
TAMEHACHI NAMIKOSHI, NARUYA TOMITA, MINORU SATOH, TAKEO SAKUTA, ATSUNORI KUWABARA, \\ HAJIME NAGASU, DAISUKE YORIMITSU, TAMAKI SASAKI and NAOKI KASHIHARA
}

Division of Nephrology, Department of Internal Medicine, Kawasaki Medical School, Kurashiki 701-0192, Japan

Received December 17, 2008; Accepted March 5, 2009

DOI: 10.3892/mmr_00000117

\begin{abstract}
High dietary protein (HP) intake is a risk factor for chronic kidney disease (CKD). HP intake is associated with the development of albuminuria and glomerulosclerosis in uninephrectomized rats. In such rats, we investigated whether HP intake induces endothelial dysfunction. Male Wistar rats were divided into sham-operated rats fed a standard-protein diet, sham-operated rats fed a high-protein diet, uninephrectomized rats fed a standard-protein diet $(\mathrm{NxSP})$ and uninephrectomized rats fed a high-protein diet (NxHP) ( $\mathrm{n}=8$ each). One week after treatment, endothelial function and urinary albumin excretion (UAE) were measured. Protein expression, phosphorylation at serine residue 1177 and uncoupling of endothelial nitric oxide synthase (eNOS), and mRNA expression of NADPH oxidase components were assessed in the aorta. NxHP rats showed hypertriglyceridemia and modest hyperhomocysteinemia. Endothelial function was significantly lower, and UAE was significantly higher in NxHP rats compared with the other groups $(\mathrm{P}<0.01$ each), although there was no difference in creatinine clearance between NxSP and NxHP rats. Expression levels, phosphorylation and the dimer/monomer ratio of eNOS did not differ among the four groups. HP intake did not modify p22phox and p47phox expression levels in uninephrectomized rats. In conclusion, HP intake induced endothelial dysfunction and enhanced albuminuria in uninephrectomized rats, independent of renal function, suggesting that HP intake may cause the development of cardiovascular disease associated with CKD.
\end{abstract}

\section{Introduction}

The reduced estimated glomerular filtration rate and microalbuminuria noted in chronic kidney disease (CKD) are risk factors for the development of cardiovascular disease (CVD)

Correspondence to: Dr Naruya Tomita, Division of Nephrology, Department of Internal Medicine, Kawasaki Medical School, 577 Matsushima, Kurashiki 701-0192, Japan

E-mail: tomita@med.kawasaki-m.ac.jp

Key words: albuminuria, chronic kidney disease, dietary protein, endothelial dysfunction, uninephrectomy
(1). This is probably due to associated endothelial dysfunction $(2,3)$. Several pathogenic mechanisms causing endothelial dysfunction have been reported, such as hypercholesterolemia, hyperglycemia, hyperhomocysteinemia and asymmetric dimethylarginine (ADMA) (4).

High dietary protein (HP) intake is considered a risk factor for the development of CKD (5). In uninephrectomized rats, chronic intake of HP results in the development of albuminuria and glomerulosclerosis through glomerular hyperfiltration or hypercholesterolemia $(6,7)$. Our previous studies indicated that HP intake induces not only renal injury in obese rats but also endothelial dysfunction $(8,9)$. However, it remains to be validated whether HP intake induces endothelial dysfunction in uninephrectomized rats, an animal model of CKD.

The aim of the present study was to determine whether HP intake induces endothelial dysfunction in uninephrectomized rats and to investigate the causative mechanisms of such dysfunction. Since endothelial dysfunction is caused by endothelial nitric oxide synthase (eNOS) dysfunction or decreased nitric oxide bioavailability through enhanced superoxide anion production (10), the protein expression, phosphorylation at serine residue 1177 and uncoupling of eNOS, and expression levels of nicotinamide adenine dinucleotide phosphate (NADPH) oxidase components in the aorta, were also examined in this study.

\section{Materials and methods}

Animals. The Ethics Review Committee for the Animal Experimentation of Kawasaki Medical School, Kurashiki, Japan, approved the experimental protocol of this study (no. 07-068). Male Wistar rats, purchased from Japan SLC (Shizuoka, Japan), were housed in a temperature- and humidity-controlled room with a 12/12 h light/dark cycle and free access to water. The rats were 9 weeks of age and were fed either standard-protein or high-protein chow up to the end of the study. Table I lists the ingredients of the standardprotein chow (MF; Oriental Yeast, Osaka, Japan) and highprotein chow (prepared by modifying AIN-93G; Oriental Yeast). One week after the initiation of the special diets, the rats were anesthetized with an intraperitoneal injection of sodium pentobarbital (40 mg/kg body weight), then either sham-operated or uninephrectomized by the removal of the right kidney. Sham-operated rats were fed either standardprotein chow (ShamSP, $n=8$ ), or high-protein chow (ShamHP, $\mathrm{n}=8$ ). Uninephrectomized rats were fed either standard-protein 
Table I. Content of standard-protein and high-protein chows.

\begin{tabular}{lcc}
\hline Ingredients & Standard protein & High protein \\
\hline Protein $(\mathrm{g} / 100 \mathrm{~g})$ & 23.6 & 42.4 \\
Fat $(\mathrm{g} / 100 \mathrm{~g})$ & 5.3 & 7.2 \\
Ash $(\mathrm{g} / 100 \mathrm{~g})$ & 6.1 & 3.6 \\
Fiber $(\mathrm{g} / 100 \mathrm{~g})$ & 2.9 & 8.0 \\
Carbohydrates $(\mathrm{g} / 100 \mathrm{~g})$ & 54.4 & 27.2 \\
Gross energy $(\mathrm{kcal} / 100 \mathrm{~g})$ & 360.0 & 343.0 \\
\hline
\end{tabular}

chow $(\mathrm{NxSP}, \mathrm{n}=8)$ or high-protein chow $(\mathrm{NxHP}, \mathrm{n}=8)$. One week after the operation (two weeks after the initiation of the special diets), rats of each group were sacrificed by an intraperitoneal injection of sodium pentobarbital $(50 \mathrm{mg} / \mathrm{kg}$ body weight). The kidneys and aorta were removed from each rat for histological and biochemical analysis. The abdominal aorta was frozen in liquid nitrogen and stored at $-80^{\circ} \mathrm{C}$, and the left kidney was fixed in $4 \%$ paraformaldehyde and embedded in paraffin.

Physiological and biochemical measurements. Systolic arterial blood pressure (SBP) was measured in pre-warmed rats by the tail-cuff method (BP-98A; Softron, Tokyo) (11). For the collection of urine samples, rats were placed in metabolic cages for $24 \mathrm{~h}$ and provided tap water but no food. Serum and urinary creatinine, fasting blood glucose (FBG), serum total cholesterol and triglyceride levels were measured at the end of the study. Serum homocysteine levels were measured by high-performance liquid chromatography (Fushimi Pharmaceutical, Kagawa, Japan). Urinary albumin excretion (UAE) was measured using an enzyme-linked immunosorbent assay kit (Exocell, Philadelphia, PA).

Morphological studies. Sections (4- $\mu \mathrm{m})$ were prepared from renal tissues embedded in paraffin and stained with periodic acid-Schiff. Morphological changes in glomeruli were assessed and photographed using a Nikon Eclipse E800 Microscope (Nikon, Tokyo) and digitalized into 1372 x 1024 pixel color scale images using the Nikon ACT-1C DXM 1200C program (Nikon).

Endothelium-dependent vascular responses. Endotheliumdependent vascular responses were measured after the preparation of rat thoracic aortic rings as described previously $(8,9)$. Briefly, a 3-mm-long cylindrical segment was cut from the aorta and bathed in $5 \mathrm{ml}$ of Krebs bicarbonate saline equilibrated with $95 \% \mathrm{O}_{2}$ and $5 \% \mathrm{CO}_{2}$, and maintained at $37^{\circ} \mathrm{C}$. Aortic segments were suspended under $1 \mathrm{~g}$ of tension and preconstricted by the addition of $3 \times 10^{-7} \mathrm{~mol} / 1$ norepinephrine. After the contraction force reached a plateau, acetylcholine, an endothelium-dependent vasodilator, was added in incremental concentrations $\left(10^{-9}\right.$ to $\left.10^{-5} \mathrm{~mol} / \mathrm{l}\right)$ to the bath. The force of isometric contraction was measured using a force-displacement transducer (model MTOB-1Z; Labo Support, Osaka). Responses to acetylcholine were expressed as a percentage of the precontracted tension induced by norepinephrine.
Western blot analysis. Western blotting was performed to detect eNOS protein and phosphorylation at serine residue 1177 (p-eNOS) in the aorta, as described previously (12). At protein extraction, complete EDTA-free and PhosSTOP (Roche Applied Science, Mannheim, Germany) were used for the inhibition of protease and phosphatase, respectively. Extracted proteins (100 $\mu \mathrm{g} /$ lane) were resolved by $7.5 \%$ sodium dodecyl sulfate-polyacrylamide gel electrophoresis (SDS-PAGE) and then electrotransferred onto polyvinylidene difluoride membranes (Millipore, Bedford, MA). After blocking, membranes were incubated with rabbit anti-eNOS, rabbit anti-p-eNOS or goat anti- $\alpha$-tubulin polyclonal antibodies (Santa Cruz Biotechnology, Santa Cruz, CA). After washing, they were incubated with horseradish peroxidaseconjugated donkey anti-rabbit IgG or anti-goat IgG (Santa Cruz Biotechnology). Resolved proteins were visualized using an enhanced chemiluminescence method (ECL Plus; Amersham Biosciences). The relative optical densities of the bands were quantified using NIH Image analysis software V1.61 (National Institutes of Health, Bethesda, MD). For the detection of the dimeric form of eNOS, samples were not heated, and the temperature of gels was maintained below $15^{\circ} \mathrm{C}$ during electrophoresis (low-temperature SDS-PAGE) as described previously (13).

Real-time quantitative PCR. RNA isolation and real-time quantitative PCR was performed as described previously (8). Briefly, total RNA was extracted from the aorta with TRIzol (Invitrogen Japan, Tokyo). The reverse transcriptase reaction was performed using a Ready-To-Go T-Primed First-Strand Kit (Amersham Biosciences, Piscataway, NJ). Real-time quantitative PCR was performed on an ABI PRISM 7700 Sequence Detection System (Applied Biosystems, Foster City, $\mathrm{CA}$ ). Primers and probes used for the p22phox and $\mathrm{p} 47 \mathrm{phox}$ were identical to those described previously (13). Changes in expression levels were quantified by standardization against RNA levels corrected for glyceraldehyde-3-phosphate dehydrogenase expression in the sample.

Statistical analysis. Values were expressed as the mean \pm SEM. Differences between groups were examined for statistical significance using the two-tailed unpaired Welch's t-test or Mann-Whitney U test, while differences among several groups were examined by one-way analysis of variance (ANOVA). A P-value $<0.05$ was considered statistically significant.

\section{Results}

Physiological and biochemical parameters. Table II summarizes the physiological and biochemical data at the end of the study. Body weight was decreased, and left kidney weight/ body weight was significantly increased in uninephrectomized rats compared with sham-operated rats $(\mathrm{P}<0.01)$. These changes did not differ between the NxSP and NxHP groups. SBP was higher in the high-protein diet groups than in the standard-protein diet groups. FBG was similar in the four groups. The serum triglyceride level was significantly higher in the NxHP group than in the ShamSP and NxSP groups $(\mathrm{P}<0.05$ each), while the serum cholesterol level was higher 
Table II. Physiological and biochemical parameters measured at the end of the study.

\begin{tabular}{|c|c|c|c|c|}
\hline & ShamSP & ShamHP & NxSP & NxHP \\
\hline Body weight (g) & $239 \pm 4$ & $235 \pm 5$ & $228 \pm 4$ & $226 \pm 5$ \\
\hline Left kidney weight/body weight (\%) & $0.46 \pm 0.02$ & $0.48 \pm 0.01$ & $0.57 \pm 0.02^{\mathrm{a}}$ & $0.59 \pm 0.01^{\mathrm{a}}$ \\
\hline Systolic blood pressure (mmHg) & $123 \pm 3$ & $133 \pm 2$ & $123 \pm 3$ & $132 \pm 5$ \\
\hline Fasting blood glucose (mg/dl) & $148 \pm 6$ & $159 \pm 6$ & $164 \pm 13$ & $162 \pm 7$ \\
\hline Serum total cholesterol $(\mathrm{mg} / \mathrm{dl})$ & $47 \pm 2$ & $47 \pm 2$ & $54 \pm 2^{\mathrm{b}}$ & $53 \pm 2$ \\
\hline Serum triglyceride $(\mathrm{mg} / \mathrm{dl})$ & $42 \pm 4$ & $50 \pm 6$ & $39 \pm 7$ & $70 \pm 8^{c}$ \\
\hline Serum homocysteine $(\mu \mathrm{mol} / \mathrm{l})$ & $12.6 \pm 0.9$ & $11.6 \pm 0.8$ & $12.0 \pm 0.5$ & $13.9 \pm 0.8$ \\
\hline
\end{tabular}

ShamSP, sham-operated rats fed standard-protein chow; ShamHP, sham-operated rats fed high-protein chow; NxSP, uninephrectomized rats fed standard-protein chow; NxHP, uninephrectomized rats fed high-protein chow. Data are expressed as the mean \pm SEM of 8 rats in each group. ${ }^{\mathrm{a} P}<0.01 ;{ }^{\mathrm{b}} \mathrm{P}<0.05$ vs. ShamSP and ShamHP; ${ }^{\mathrm{c}}<0.05$ vs. ShamSP and NxSP.

A

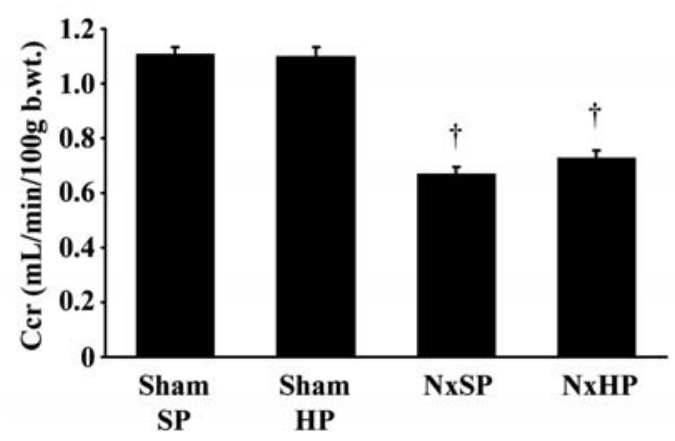

B

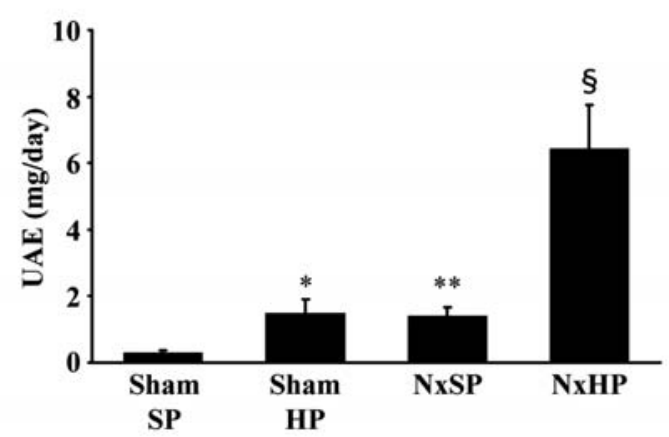

Figure 1. Creatinine clearance (A) and urinary albumin excretion (B) at the end of the study. Creatinine clearance (Ccr) was significantly lower in the NxSP and NxHP groups compared with the ShamSP and ShamHP groups. It was not different between the NxSP and NxHP groups. Urinary albumin excretion (UAE) was significantly higher in the ShamHP and NxSP groups compared with the ShamSP group. It was significantly higher in the NxHP group compared with the other three groups. Data are mean $\pm \mathrm{SEM}$ of 8 rats in each group. ${ }^{*} \mathrm{P}<0.05,{ }^{* *} \mathrm{P}<0.01$ vs. ShamSP, ${ }^{\dagger} \mathrm{P}<0.01$ vs. ShamSP and ShamHP, ${ }^{\S} \mathrm{P}<0.01$ vs. ShamSP, ShamHP and NxSP. b.wt., body weight.

in uninephrectomized rats than in sham-operated rats. The serum homocysteine level tended to be higher in the NxHP group than in the other three groups, albeit insignificantly. Of note, the NxHP group showed a significantly higher serum triglyceride level and a modestly higher serum homocysteine level compared with the NxSP group.

Creatinine clearance and urinary albumin excretion. Creatinine clearance was significantly lower in the NxSP $(0.67 \pm 0.02 \mathrm{ml} / \mathrm{min} / 100 \mathrm{~g}$ body weight, $\mathrm{P}<0.01)$ and NxHP $(0.73 \pm 0.03, \mathrm{P}<0.01)$ groups compared with the ShamSP $(1.11 \pm 0.03)$ and ShamHP $(1.10 \pm 0.03)$ groups, respectively, although it did not differ significantly between the NxSP and NxHP groups (Fig. 1A). UAE was significantly higher in the ShamHP $(1.49 \pm 0.41 \mathrm{mg} / \mathrm{day}, \mathrm{P}<0.05)$ and $\mathrm{NxSP}$ $(1.41 \pm 0.26, \mathrm{P}<0.01)$ groups than in the ShamSP group $(0.30 \pm 0.07)$ (Fig. 1B), and was significantly higher in the NxHP group $(6.43 \pm 1.31 \mathrm{mg} /$ day, $\mathrm{P}<0.01)$ compared with the ShamSP, ShamHP and NxSP groups. These results indicate that HP intake enhanced albuminuria, independent of renal function.
Morphological studies. Glomerular hypertrophy was observed in the ShamHP (Fig. 2B), NxSP (Fig. 2C) and NxHP groups (Fig. 2D) compared with the ShamSP group (Fig. 2A), although it did not apparently differ between the first three groups. No other morphological abnormalities were detected in the glomeruli in all four groups.

Endothelium-dependent vascular responses. The relaxation response was significantly lower in the NxHP group compared with the ShamSP and NxSP groups at $10^{-7}$ to $10^{-5} \mathrm{~mol} / \mathrm{l}$ acetylcholine $(\mathrm{P}<0.01)$ and compared with the ShamHP group at $10^{-6}$ and $10^{-5} \mathrm{~mol} / 1$ acetylcholine $(\mathrm{P}<0.01$, Fig. 3$)$. These results suggest that HP intake resulted in the deterioration of the endothelium-dependent vascular responses of the aorta in uninephrectomized rats, independent of renal function.

Western blot analysis. Protein expression levels of eNOS, p-eNOS and dimeric eNOS in the aorta are shown in Fig. 4. Bands for eNOS and the dimeric form were detected at 130 and $260 \mathrm{kDa}$, respectively (Fig. 4A). Protein expression levels of eNOS, p-eNOS and dimeric eNOS were evaluated as the 

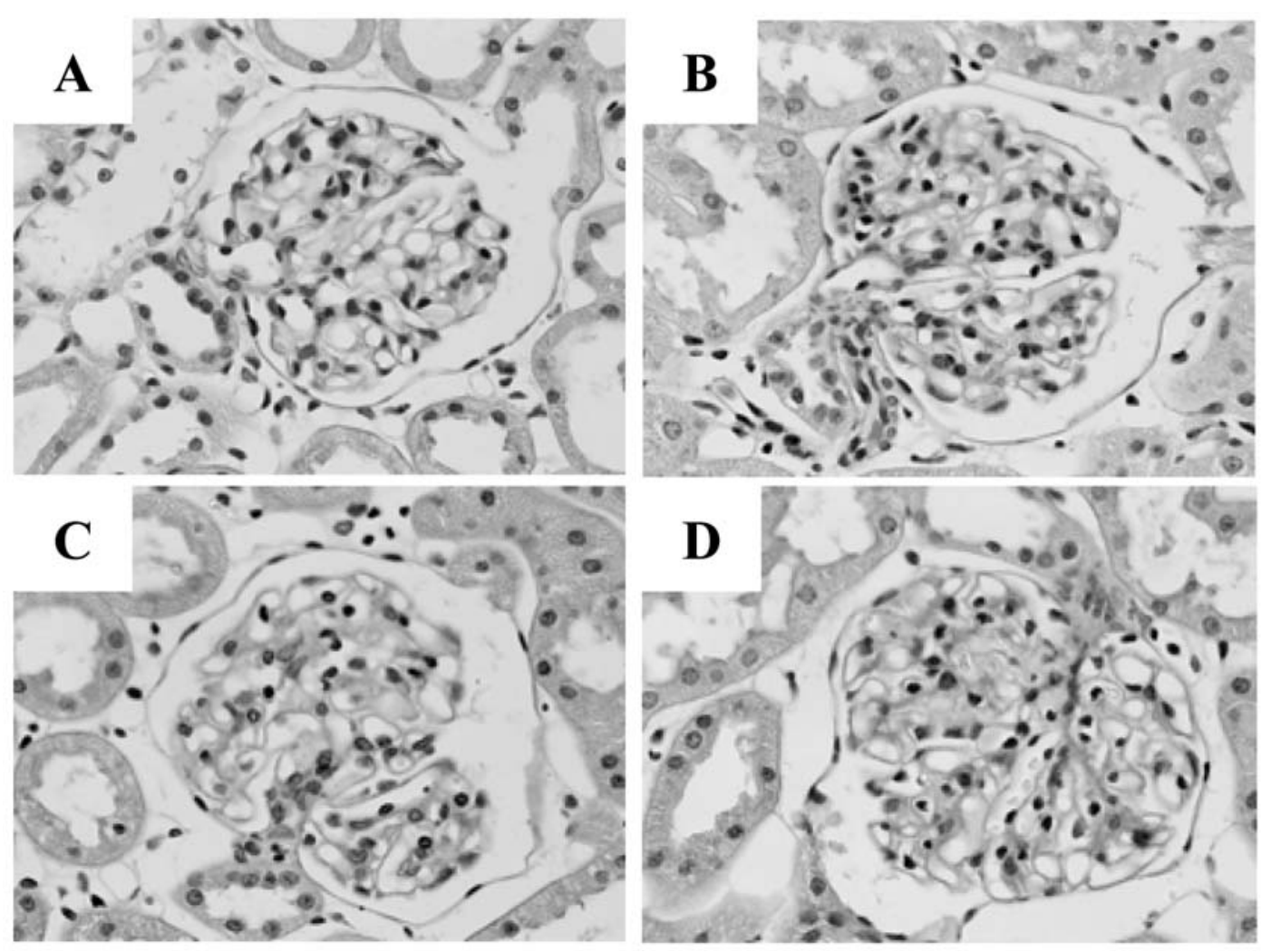

Figure 2. Morphological changes in glomeruli stained with periodic acid-Schiff. Glomerular hypertrophy was observed in the ShamHP (B), NxSP (C) and NxHP (D) groups compared with the ShamSP group (A). Glomerular size did not apparently differ among the first three groups. No other glomerular morphological abnormalities were detected in any of the four groups. Original magnification, $\mathrm{x} 400$.

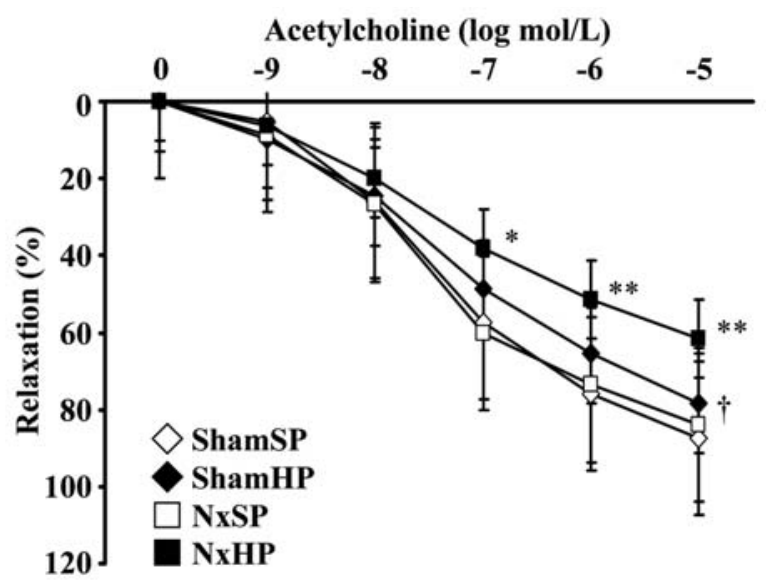

Figure 3. Endothelium-dependent vascular responses of the aorta to acetylcholine. Relaxation responses were significantly lower in the NxHP group compared with the ShamSP and NxSP groups at $10^{-7}$ to $10^{-5} \mathrm{~mol} / 1$ acetylcholine and compared with the ShamHP group at $10^{-6}$ and $10^{-5} \mathrm{~mol} / \mathrm{l}$ acetylcholine. Data are the mean \pm SEM of 6 or 7 rats in each group. ${ }^{*} \mathrm{P}<0.01$ vs. ShamSP and NxSP, ${ }^{* *} \mathrm{P}<0.01$ vs. ShamSP, ShamHP and NxSP, ${ }^{\dagger} \mathrm{P}<0.05$ vs. ShamSP.

eNOS/ $\alpha$-tubulin ratio (Fig. 4B), p-eNOS/eNOS ratio (Fig. 4C) and dimer/monomer ratio (Fig. 4D), respectively. Expression levels of all test proteins did not differ among the four groups. These results indicate that HP intake and uninephrectomy did not modify eNOS expression, phosphorylation at serine residue 1177 or uncoupling within the 2 -week period of the study.
Real-time quantitative PCR. mRNA expression levels of both p22phox (Fig. 5A) and p47phox (Fig. 5B) in the aorta of the NxSP and NxHP groups were not significantly different, indicating that HP intake did not increase the mRNA expression levels of NADPH oxidase components in uninephrectomized rats within the 2-week period of the study.

\section{Discussion}

In the present study, we demonstrated that HP intake induced endothelial dysfunction and enhanced albuminuria in uninephrectomized rats, independent of renal function. It was accompanied with significantly higher serum triglyceride levels and modest increases in serum homocysteine levels. On the other hand, HP intake did not modify eNOS expression, phosphorylation at serine residue 1177 and uncoupling, and did not increase expression levels of NADPH oxidase components in uninephrectomized rats. The results suggest that HP intake caused endothelial dysfunction in uninephrectomized rats independent of eNOS and NADPH oxidase expression levels.

Increased serum levels of triglyceride and homocysteine may be associated with endothelial dysfunction and enhanced albuminuria in uninephrectomized rats fed a high-protein diet. Impaired endothelium-dependent vasodilatation in the forearms was reported in patients with hypertriglyceridemia $(14,15)$. Transient triglyceridemia induced by the infusion of a triglyceride emulsion also decreased flow-induced vasodilatation in healthy young men (16). However, it is not clear whether hypertriglyceridemia directly enhances albuminuria, although the former was reported to be independently 
A

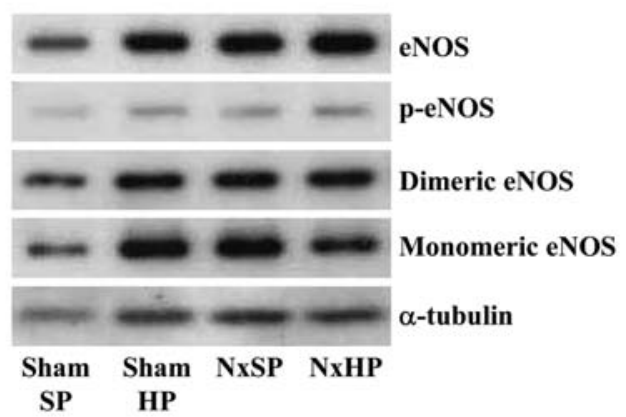

C

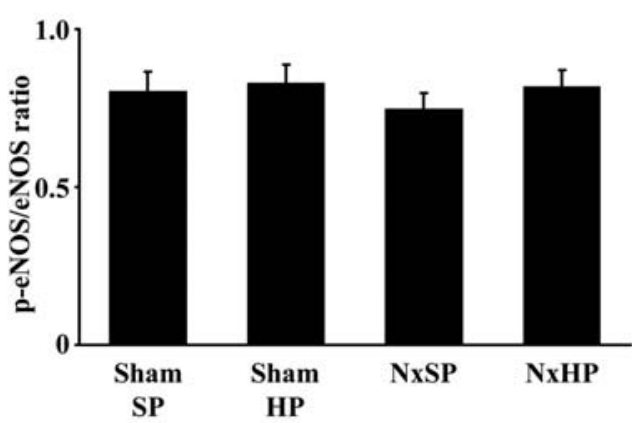

B

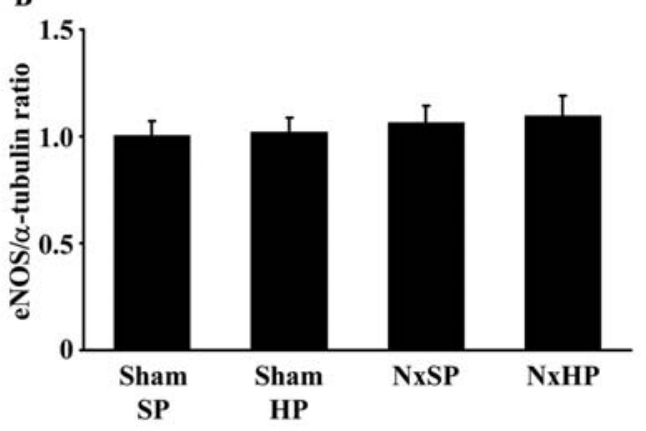

D

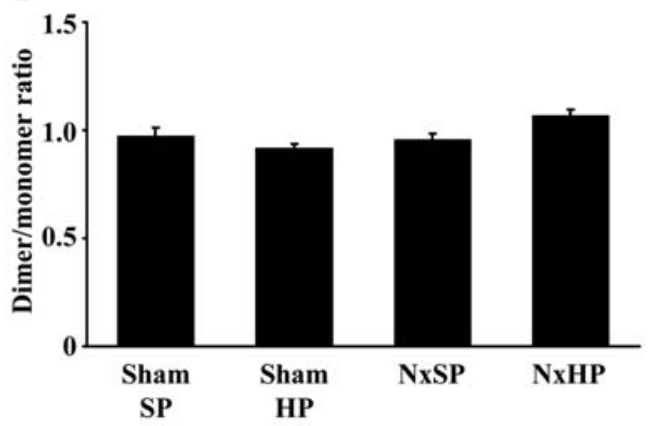

Figure 4. Western blot analysis for endothelial nitric oxide synthase (eNOS), phosphorylation at serine residue 1177 (p-eNOS) and dimeric eNOS in the aorta. Bands for eNOS and the dimeric form were detected at 130 and $260 \mathrm{kDa}$, respectively (A). Protein expression levels of eNOS, p-eNOS and dimeric eNOS were evaluated as the eNOS/ $\alpha$-tubulin ratio (B), p-eNOS/eNOS ratio (C) and dimer/monomer ratio (D), respectively. The expression levels of the proteins did not differ among the four groups. Data are the mean \pm SEM of 6 rats.

A

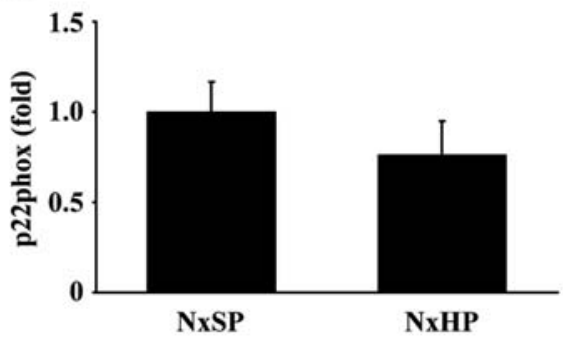

B

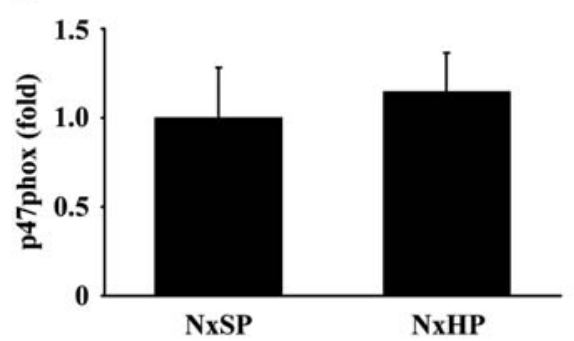

Figure 5. Real-time PCR for p22phox (A) and p47phox (B) in the aorta. mRNA expression levels of both p22phox and p47phox did not differ significantly between the NxSP and NxHP groups. Data are the mean \pm SEM of 8 rats.

associated with the prevalence of albuminuria in a diabetes population (17). On the other hand, small physiological increments in plasma homocysteine induced by dietary protein intake were reported to reduce flow-mediated vasodilatation in the brachial arteries (18). A cross-sectional clinical study suggested that plasma homocysteine could be an intermediate factor in the relationship between endothelial dysfunction and renal function (19). Intriguingly, acetylcysteine improved flow-mediated vasodilatation in the forearm, reducing plasma homocysteine concentration in patients with end-stage renal failure (20). In addition, the Hoorn Study revealed that hyperhomocysteinemia and dietary protein intake were related to microalbuminuria independent of type 2 diabetes mellitus and hypertension (21). In the general population, hyperhomocysteinemia was reported to be a significant risk factor for renal insufficiency (22), while renal insufficiency was strongly associated with an increased risk of hyperhomocysteinemia (23).

HP intake did not alter eNOS expression, phosphorylation at serine residue 1177 and uncoupling, and expression levels of NADPH oxidase components in the aorta of uninephrectomized rats. A previous study reported the presence of high plasma levels of ADMA and reduced endotheliumdependent vasodilatation of coronary arteries in dogs four weeks after uninephrectomy (24). In the present study, however, endothelium-dependent vascular responses of the aorta were not different between the ShamSP and NxSP groups, one week after uninephrectomy. Further studies are needed to elucidate the pathogenic mechanisms of dietary protein-induced endothelial dysfunction in renal insufficiency.

In conclusion, in the present study we demonstrated that HP intake induced endothelial dysfunction and enhanced 
albuminuria in uninephrectomized rats, an animal model of CKD. These changes were accompanied by hypertriglyceridemia and a modest increase in serum homocysteine, which are possibly involved in the development of endothelial dysfunction and albuminuria. These results suggest that the restriction of dietary protein intake is important in preventing CVD in patients with CKD.

\section{Acknowledgements}

We thank Ms. Satomi Hanada, Ms. Etsuko Yorimasa and Ms. Asuka Maeda for the excellent technical assistance. This work was supported by a Grant-in-Aid for Young Scientists (Start-up) from the Japan Society for the Promotion of Science (no. 20890271), and by a Research Project Grant from Kawasaki Medical School (no. 20-3030).

\section{References}

1. McCullough PA, Jurkovitz CT, Pergola PE, et al: Independent components of chronic kidney disease as a cardiovascular risk state: results from the Kidney Early Evaluation Program (KEEP). Arch Intern Med 167: 1122-1129, 2007.

2. Stam F, van Guldener C, Becker A, Dekker JM, Heine RJ, Bouter LM and Stehouwer CDA: Endothelial dysfunction contributes to renal function-associated cardiovascular mortality in a population with mild renal insufficiency: the Hoorn Study. J Am Soc Nephrol 17: 537-545, 2006.

3. Ochodnicky P, Henning RH, van Dokkum RPE and de Zeeuw D: Microalbuminuria and endothelial dysfunction: emerging targets for primary prevention of end-organ damage. J Cardiovasc Pharmacol 47 (Suppl 2): 151-162, 2006.

4. Goligorsky MS: Endothelial cell dysfunction: can't live with it, how to live without it. Am J Physiol Renal Physiol 288: F871-F880, 2005.

5. Taal MW and Brenner BM: Predicting initiation and progression of chronic kidney disease: Developing renal risk scores. Kidney Int 70: 1694-1705, 2006.

6. Hostetter TH, Meyer TW, Rennke HG and Brenner BM: Chronic effects of dietary protein in the rat with intact and reduced renal mass. Kidney Int 30: 509-517, 1986.

7. O'Donnell MP, Kasiske BL, Schmitz PG and Keane WF: High protein intake accelerates glomerulosclerosis independent of effects on glomerular hemodynamics. Kidney Int 37: 1263-1269, 1990.

8. Namikoshi T, Satoh M, Tomita N, et al: Pioglitazone ameliorates endothelial dysfunction in obese rats with nephropathy. Biochem Biophys Res Commun 361: 835-840, 2007.

9. Namikoshi T, Tomita N, Satoh M, et al: Olmesartan ameliorates renovascular injury and oxidative stress in Zucker obese rats enhanced by dietary protein. Am J Hypertens 10: 1085-1091, 2007.
10. Förstermann U and Münzel T: Endothelial nitric oxide synthase in vascular disease: from marvel to menace. Circulation 113: 1708-1714, 2006.

11. Tomita N, Higaki J, Kaneda Y, Yu H, Morishita R, Mikami H and Ogihara T: Hypertensive rats produced by in vivo introduction of the human renin gene. Circ Res 73: 898-905, 1993.

12. Namikoshi T, Tomita N, Fujimoto S, et al: Isohumulones derived from hops ameliorate renal injury via an anti-oxidative effect in Dahl salt-sensitive rats. Hypertens Res 30: 175-184, 2007.

13. Satoh M, Fujimoto S, Haruna Y, et al: $\mathrm{NAD}(\mathrm{P}) \mathrm{H}$ oxidase and uncoupled nitric oxide synthase are major sources of glomerular superoxide in rats with experimental diabetic nephropathy. Am J Physiol Renal Physiol 288: F1144-F1152, 2005.

14. Lewis TV, Dart AM and Chin-Dusting JPF: Endotheliumdependent relaxation by acetylcholine is impaired in hypertriglyceridemic humans with normal levels of plasma LDL cholesterol. J Am Coll Cardiol 33: 805-812, 1999.

15. Lundman P, Eriksson MJ, Stühlinger M, Cooke JP, Hamsten A and Tornvall P: Mild-to-moderate hypertriglyceridemia in young men is associated with endothelial dysfunction and increased plasma concentrations of asymmetric dimethylarginine. J Am Coll Cardiol 38: 111-116, 2001.

16. Lundman P, Eriksson M, Schenck-Gustafsson K, Karpe F and Tornvall P: Transient triglyceridemia decreases vascular reactivity in young, healthy men without risk factors for coronary heart disease. Circulation 96: 3266-3268, 1997.

17. Young BA, Katon WJ, von Korff M, et al: Racial and ethnic differences in microalbuminuria prevalence in a diabetes population: the Pathways Study. J Am Soc Nephrol 16: 219-228, 2005.

18. Chambers JC, Obeid OA and Kooner JS: Physiological increments in plasma homocysteine induce vascular endothelial dysfunction in normal human subjects. Arterioscler Thromb Vasc Biol 19: 2922-2927, 1999.

19. Stam F, van Guldener C, Schalkwijk CG, ter Wee PM, Donker AJM and Stehouwer CDA: Impaired renal function is associated with markers of endothelial dysfunction and increased inflammatory activity. Nephrol Dial Transplant 18: 892-898, 2003.

20. Scholze A, Rinder C, Beige J, Riezler R, Zidek W and Tepel M: Acetylcysteine reduces plasma homocysteine concentration and improves pulse pressure and endothelial function in patients with end-stage renal failure. Circulation 109: 369-374, 2004.

21. Hoogeveen EK, Kostense PJ, Jager A, et al: Serum homocysteine level and protein intake are related to risk of microalbuminuria: the Hoorn Study. Kidney Int 54: 203-209, 1998.

22. Ninomiya T, Kiyohara Y, Kubo M, et al: Hyperhomocysteinemia and the development of chronic kidney disease in a general population: the Hisayama Study. Am J Kidney Dis 44: 437-445, 2004.

23. Francis ME, Eggers PW, Hostetter TH and Briggs JP: Association between serum homocysteine and markers of impaired kidney function in adults in the United States. Kidney Int 66: 303-312, 2004.

24. Tatematsu S, Wakino S, Kanda T, et al: Role of nitric oxideproducing and -degrading pathways in coronary endothelial dysfunction in chronic kidney disease. J Am Soc Nephrol 18: 741-749, 2007. 